\title{
GENERATION OF TURBULENT INFLOW CONDITIONS FOR LARGE EDDY SIMULATION FROM STEREOSCOPIC PIV MEASUREMENTS
}

\author{
Laurent Perret, Joël Delville, Rémi Manceau and Jean-Paul Bonnet \\ Laboratoire d'Etudes Aérodynamiques - UMR CNRS 6609 \\ Université de Poitiers - ENSMA \\ C.E.A.T., 43, route de l'Aérodrome F-86036 Poitiers - France
}

\begin{abstract}
A novel method to generate inflow data for unsteady numerical simulations is proposed. This method consists in coupling an experimental database obtained by stereoscopic PIV measurements which are under-resolved in time to a numerical code. Based on the Proper Orthogonal Decomposition of this database, the proposed approach enables the adaptation of the experimental mesh to the grid of the simulation and the modelling of the temporal dynamics of the flow field in the inlet section by gaussian random time series that present the correct one- and two- point statistics. To test this method, a LES of a turbulent plane mixing layer configuration is performed.
\end{abstract}

\section{INTRODUCTION}

The prescription of well-suited boundary conditions is a crucial issue when performing unsteady numerical computations of turbulent flows. In the case of the simulation of a spatially developing flow, the specification of the flow field at the inlet of the computational domain conditions the downstream development of the flow. The simplest and historical method to adress this problem is to impose well chosen infinitesimal perturbations, where intrinsic most amplified modes are present, on a laminar mean flow profile at the inflow section and to let the flow evolve to a fully turbulent state. The main drawback of this approach is the need of a large computational domain required for the transition to the turbulent state to occur. Hence, the computational cost of the simulation can be dramatically increased. Consequently, several studies (see Keating et al. (2004) for a detailed review) have been devoted to design more efficient methods to prescribe realistic turbulent inflow conditions which present correct mean values, proper one-point and two-point correlations and given spectrum as well as correct phase information. This can be achieved by running a separate precursor calculation ( $\mathrm{Li}$ et al., 2000) from which a velocity field in a plane normal to the streamwise direction is extracted. This sequence of planes is used afterward as inlet conditions for the main computation. To avoid the additionnal cost of the precursor simulation, Lund et al. (1998) proposed a recyling method which consists in extracting velocity data from a downstream section, rescaling the velocity profile to reintroduce them at the inlet section of the domain. Nevertheless, this method involves the existence of a region where similarity laws enable the velocity profiles to be rescaled. Moreover, the periodicity effect of this recyling procedure can lead to the excitation of some particular modes. An alternative to these methods is to use synthetic turbulence generated by using random numbers satisfying con- straints e.g. first and second order moments and spectra (Lee et al., 1992). However, simulations using these approaches require an adaptation zone downstream of the inlet section, due to the unphysical random character of the synthetized velocity fields. Moreover, correct target statistics, especially two-point information, can be difficultly obtained. Recently, Druault et al. (2004) developed a method which enables the unsteady coupling between an experimental database obtained through hot-wire measurements and a spatially-developing Large Eddy Simulation (LES). This technique was based on the joint use of the Proper Orthogonal Decomposition (POD) and the Linear Stochastic Estimation (LSE), permitting the reconstruction of the velocity field on the full mesh of the inlet section from only a few measurement locations. Although this method takes advantage of the good temporal resolution of hot-wire measurements, it suffers from a low spatial resolution due to the limited number of probes that can be implemented simultaneously.

The goal of the proposed method, based on the use of the POD, is to couple a database obtained by Stereoscopic Particle Image Velocimetry (SPIV) to a computational code. This measurement technique provides a spatial resolution consistent with that of the numerical simulation. To compensate the low temporal resolution offered by the SPIV measurements, the temporal behaviour of the flow in the inlet section is modelled by synthetic random time series.

In the present paper, this method is used to perform a LES of a turbulent plane mixing layer, the inlet section of the computational domain being located at the beginning of the self-similarity region.

\section{PRINCIPLES}

The proposed inlet condition generation method aims at interfacing experimental data which are under-resolved in time to an unsteady computational code. Once the database is acquired, the method consists of two steps. Firstly, the experimental mesh must be adapted to the numerical grid, which can imply spatial interpolation and extrapolation in the trasversal and spanwise directions, and the generated velocity fields must be consistent with the other boundary conditions retained for the simulation (namely periodic condition in the spanwise direction, free-slip conditions at the horizontal boundaries). Secondly, the temporal dynamics of the flow in the inlet section must be correctly reproduced. The POD, as described later, is used to uncouple these two steps by decomposing the velocity field into a set of spatial and temporal modes (respec- 


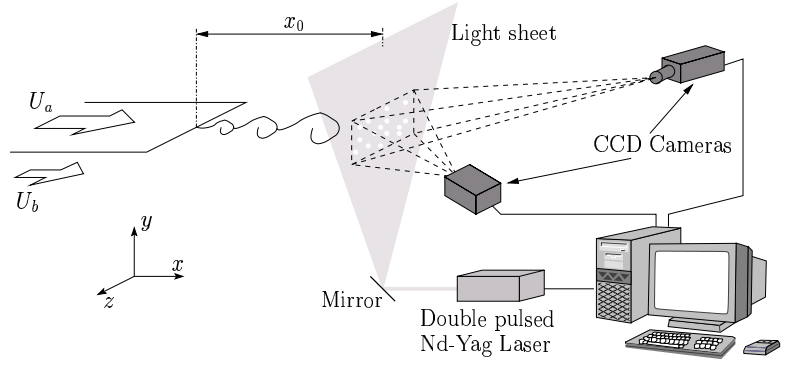

Figure 1: Experimental set up.

tively $\phi_{i}{ }^{n}(\mathbf{x})$ and $\left.a^{n}(t)\right)$ :

$$
u_{i}(\mathbf{x}, t)=\sum_{n=1}^{N_{s}} a^{(n)}(t) \phi_{i}^{(n)}(\mathbf{x})
$$

Thus, the first step is performed by independently processing each spatial eigenvector $\phi_{i}^{(n)}(\mathbf{x})$. Futhermore, the anisotropy and the inhomogeneity of the velocity field is taken into account implicitly by these spatial modes. The global dynamics of the whole flow field is represented by the temporal projection coefficients $a^{(n)}(t)$. In the present case, due to the low sampling frequency of the SPIV system compared to the characteristic frequencies of the flow, these temporal coefficients are synthetically modelled.

\section{FLOW CONFIGURATION AND EXPERIMENTAL SET UP}

An experimental database is obtained from SPIV measurements. A sketch of the experimental set up is presented in Figure 1. A plane subsonic turbulent air/air mixing layer with a high-speed velocity $U_{a}$ of $35.2 \mathrm{~m} / \mathrm{s}$ and a low-speed velocity $U_{b}$ equal to $23.8 \mathrm{~m} / \mathrm{s}$ (velocity ratio $r=0.67$ ) is used. The coordinate system is $x$ in the streamwise direction, $y$ in the vertical direction and $z$ in the spanwise direction. The origin is located at the splitter plate trailing edge. The measurements were performed in a section of the flow normal to the streamwise direction, located at $x_{0}=300 \mathrm{~mm}$ from the trailing edge of the splitter plate, corresponding to the beginning of the selfsimilarity region of the mean flow quantities. The Reynolds number, based on the mean velocity $U_{m}=\left(U_{a}+U_{b}\right) / 2$ and the vorticity thickness in the measurement section $\delta_{\omega_{0}}=18.7$ $\mathrm{mm}$, is $R e=\delta_{\omega_{0}} U_{m} / \nu \simeq 36,000$. The typical frequency $f_{c}$ associated to the large scale structures in the mixing layer, corresponding to a Strouhal number $S t=f_{c} \delta_{\omega_{0}} / U_{m}=0.3$, is about $480 \mathrm{~Hz}$.

The SPIV set up comprises two double frame CCD cameras $1350 \times 1049$ pixels $^{2}$ in angular stereoscopic configuration to record images of the flow and a double-pulsed Nd-Yag laser to create the light sheet. The flow was seeded with olive oil tracer particles. Acquisition, synchronisation and SPIV post-processing were performed with the Davis 6.2 Lavision software. More details about the flow investigated and the SPIV set up can be found in Perret (2004) and Perret et al. (2004). The size of the field of SPIV measurement, $L_{y} \times L_{z}=4 \delta_{\omega_{0}} \times 6.1 \delta_{\omega_{0}}$, is chosen large enough to capture the main scales of the flow in the $y$ - and $z$-directions while preserving a good spatial resolution of about $0.088 \delta_{\omega_{0}}$. A set of $N_{s}=2,000$ velocity fields was acquired at a frequency of 1 $\mathrm{Hz}$.
METHOD FOR EXPERIMENT/COMPUTATION INTERFACING

\section{Proper Orthogonal Decomposition}

In this section, the Proper Orthogonal Decomposition is briefly presented. More details can be found in Berkooz et al. (1993) and Sirovich (1987). Lumley (1967) first proposed the POD technique to identify the coherent structures in turbulent flows. It consists in extracting from the flow the structure $\phi(\mathbf{X})$ with the largest mean-square projection onto the velocity field $\mathbf{u}(\mathbf{X}, t)$. This maximization problem leads to the solving of the integral problem of eigenvalues:

$$
\int_{\mathcal{D}} R_{i j}\left(\mathbf{X}, \mathbf{X}^{\prime}\right) \phi_{j}^{(n)}\left(\mathbf{X}^{\prime}\right) d \mathbf{X}^{\prime}=\lambda^{(n)} \phi_{i}^{(n)}(\mathbf{X})
$$

where $\lambda^{(n)}$ corresponds to the $n$-th eigenvalue and represents the amount of energy contained in the mode $\phi^{(n)}(\mathbf{X}) . R_{i j}$ is the two-point space correlation tensor over the domain $\mathcal{D}$ :

$$
R_{i j}\left(\mathbf{X}, \mathbf{X}^{\prime}\right)=\left\langle u_{i}(\mathbf{X}, t) u_{j}\left(\mathbf{X}^{\prime}, t\right)\right\rangle
$$

where $\langle$.$\rangle is the ensemble average operator. The fluctuating$ field can be projected onto the POD basis composed of the eigenfunctions $\phi_{i}^{(n)}(X)$ :

$$
u_{i}(\mathbf{X}, t)=\sum_{n=1}^{\infty} a^{(n)}(t) \phi_{i}^{(n)}(\mathbf{X})
$$

The projection coefficients are computed as:

$$
a^{(n)}(t)=\int_{\mathcal{D}} u_{i}(\mathbf{X}, t) \phi_{i}^{(n)}(\mathbf{X}) d \mathbf{X}
$$

and are uncorrelated in time:

$$
\left\langle a^{(n)}(t) a^{(m)}(t)\right\rangle=\lambda^{(n)} \delta_{m}^{n}
$$

In the present work, we used the version proposed by Sirovich (1987), called the Snapshot POD, which is more adapted to data which are well-resolved in space with a limited number of time samples. Moreover, the spatial domain considered here is the $y-z$ plane. Consequently, the spatial eigenvectors are function of two variables: $\phi_{i}^{(n)}(y, z), i=1,2$, 3.

\section{Horizontal extrapolation}

In the homogeneous spanwise direction, a periodic condition is retained in the numerical simulation. Hence, inlet conditions must satisfy this constraint at each time step. Periodicity has then to be imposed to experimental data that are a priori non-periodic. Two steps are involved: 1) determination of the size of the domain on which the periodic condition must be imposed, 2) extrapolation and periodization of the data on this domain:

1) POD eigenvectors are known to degenerate into harmonic modes when the POD is performed in a homogeneous direction (Berkooz et al, 1993). As proposed by Coiffet et al. (2002), the POD can then be used in the spanwise direction to detect a global wave length in the data. The advantage of this approach is that the 


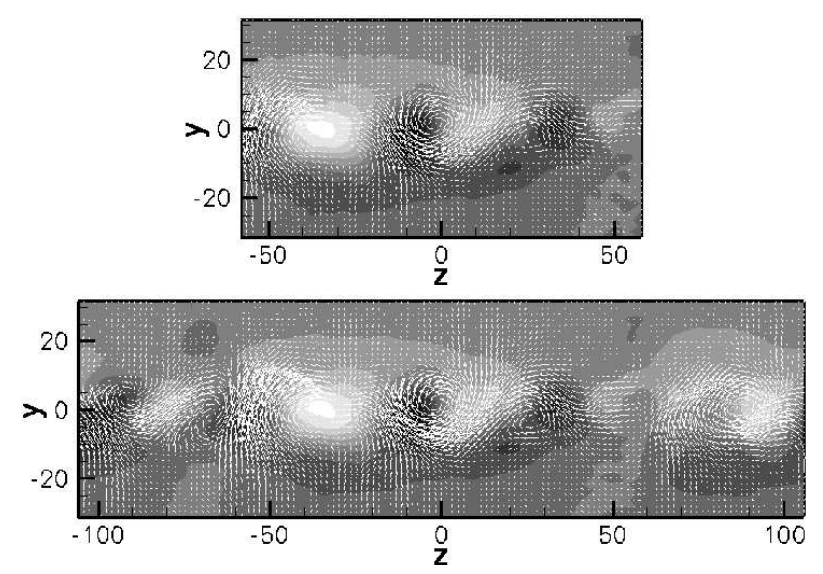

Figure 2: Example of a Snapshot POD eigenvector $\phi_{i}^{(n)}(y, z)$ (top) and its spanwise extrapolation (bottom) $(n=8)$.

wave length of the POD eigenvectors is not constrained by the size of the domain on which the POD is applied, as a Fourier transform would be. Thus, at each vertical location $y$, a one-dimensional POD is performed in the direction $z$ in order to detect a common wave length that is not directly related to the size of the measurement domain.

2) Once the spanwise extent of the periodic domain is known, each two-dimensional Snapshot POD eigenvector $\phi_{i}^{(n)}(y, z)$ is individually extrapolated and periodized. In that manner, the final reconstructed velocity field $u_{i}(\mathbf{x}, t)=\sum_{n=1}^{N_{s}} a^{(n)}(t) \phi_{i}^{(n)}(\mathbf{x})$ satisfies the periodicity condition. To perform this extrapolation, the technique of selective deconvolution, proposed by Franke (1987) to extrapolate signals, the spectrum of which shows dominant spectral frequencies, is retained. This algorithm is iterative and is based on direct and inverse fast Fourier transforms (FFT). The final signal is periodic by construction on the final domain. Moreover, this approach enables to remove the windowing effect from the signal caused by the limited extent of the measurement domain.

This extrapolation procedure is applied to the velocity fields obtained from the SPIV measurements. The first step, which consists in detecting a wave length to periodize the domain, leads to a domain which spanwise extent is 1.83 time larger than the original measurement window.

Each Snapshot POD eigenvector $\phi_{i}^{(n)}(y, z)$ is then extrapolated is the $z$-direction via a bidimensional approach of the algorithm of Franke (1987) using bidimensional FFT.

As can be seen in Figure 2, the extrapolation procedure do not introduce any spatial discontinuity in the eigenvectors and correctly spreads the motives corresponding to the turbulent structures extracted by the POD. Moreover, it can be noticed that the extrapolated eigenvectors are periodic in the final domain.

\section{Vertical extrapolation}

Conditions chosen on the upper and lower horizontal boundaries of the computational domain are free-slip conditions. Moreover, to prevent the flow from being confined in this direction, an extension of the inlet domain in the vertical

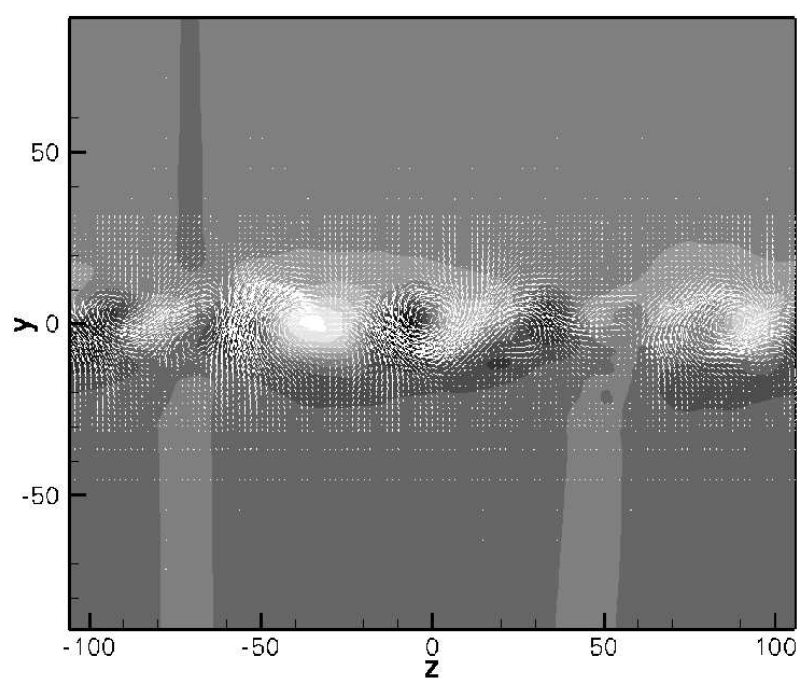

Figure 3: Vertical and spanwise extrapolation of the Snapshot POD eigenvector $\widetilde{\phi}_{i}^{(n)}(y, z)$ presented on Figure $2(n=8)$.

direction is required. Hence, the experimental velocity fields must also be extrapolated in this direction. A one-dimensional POD approach is used to extrapolate each Snapshot POD eigenvector $\phi_{i}^{(n)}(y, z)$ in the $y$-direction. Here, each spanwise location $z$ is processed independently by the following steps:

1) compute the two-point correlation tensor $R\left(y, y^{\prime}\right)=$ $\left\langle u_{i}(y) u_{i}\left(y^{\prime}\right)\right\rangle$ from the original velocity fields where $\langle$. is the average operator in both the temporal and spanwise directions.

2) perform a POD decomposition of this tensor to obtain a POD basis of $N_{y}$ one-dimensional eigenvectors $\psi_{i}^{(m)}(y)$, $N_{y}$ being the number of points in the vertical direction. The snapshot POD eigenvectors $\phi_{i}^{(n)}(y, z)$ can be projected onto the basis of the vectors $\psi_{i}^{(m)}(y)$ :

$$
\phi_{i}^{(n)}(y, z)=\sum_{m=1}^{N_{y}} c^{(m, n)}(z) \psi_{i}^{(m)}(y)
$$

3) As pointed out by Druault and Delville (2000), POD eigenvectors $\psi_{i}^{(m)}(y)$ obtained in the direction of inhomogeneity $y$ in free turbulent shear flows must decrease to zero as the energy tends to 0 when $y$ tends to $\pm \infty$. Moreover, in the case of the mixing layer, the $\psi_{i}^{(m)}(y)$ decrease exponentially. Hence, the basis $\left\{\psi_{i}^{(m)}(y)\right\}$ can be extrapolated in the $y$-direction on the final domain into a new set of modes $\left\{\widetilde{\psi}_{i}^{(m)}(y)\right\}$

4) Extrapolated bidimensional Snapshot POD eigenvectors $\widetilde{\phi}_{i}^{(n)}(y, z)$ can then be reconstructed over the larger domain using the extrapolated basis $\left\{\widetilde{\psi}_{i}^{(m)}(y)\right\}$ :

$$
\widetilde{\phi}_{i}^{(n)}(y, z)=\sum_{m=1}^{N_{y}} c^{(m, n)}(z) \widetilde{\psi}_{i}^{(m)}(y)
$$

Example of an extrapolated Snapshot POD mode in the vertical direction is shown in Figure 3. Eventually, the eigenvectors $\widetilde{\phi}_{i}^{(n)}(y, z)$ are used to reconstruct the velocity field in the final domain: $\widetilde{u}_{i}(y, z, t)=\sum_{n=1}^{N_{s}} a^{(n)}(t) \widetilde{\phi}_{i}^{(n)}(y, z)$. 

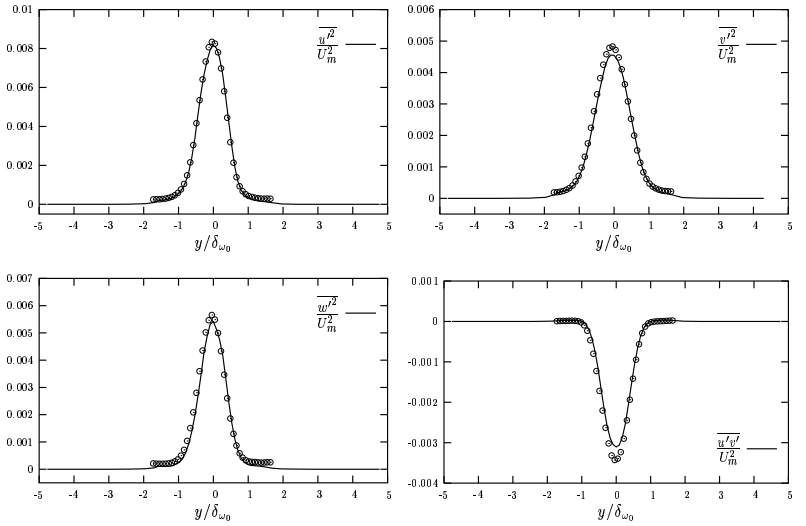

Figure 4: Reynolds stress profiles $\overline{u_{i}^{\prime} u_{j}^{\prime}} / U_{m}^{2}$ of the synthetized $(-)$ and experimental (o) velocity fields.

\section{Temporal modelling}

The temporal behaviour of the POD modes is modelled through the projection coefficients $a^{(n)}(t)$. Given the fact that no spectral information is available about these coefficients except their total energy content (e.g. the eigenvalues $\left.\lambda^{(n)}\right)$, the $a^{(n)}(t)$ are modelled by time series of random numbers. Since these coefficients are mutually uncorrelated in time (Equation 6 ), they can be generated independently. $N_{s}$ gaussian time series $g^{(n)}(t)$ of random numbers of zero mean and rms equals to 1 are first generated. Then, to enforce a realistic spectral repartition of energy, a spectral transfer function, deduced from a modified Von Karman spectrum $E_{P a o}(f)$ proposed by Pao (Hinze, 1975), is applied:

$$
\hat{a}^{(n)}(f)=\hat{g}^{(n)}(f)\left(\frac{E_{P a o}(f)}{E_{g g}(f)}\right)^{1 / 2}
$$

where $\hat{g}^{(n)}(f)$ is the Fourier transform of $g^{(n)}(t)$ and $E_{g g}(f)$ the spectrum of $g^{(n)}(t)$. Characteristic frequencies delimiting the inertial range of the velocity spectra are evaluated from prior hot-wire measurements (Perret, 2004). Random time series are finally normalized to ensure that $\left\langle a^{(n)} a^{(n)}\right\rangle=\lambda^{(n)}$. Hence, the velocity field which results from the linear combination of the different POD modes presents the correct oneand two-point spatial statistics with a spectral repartition of the energy given by the shape of the chosen theoretical spectrum.

\section{GENERATED INFLOW CONDITIONS}

A database of synthetic velocity fields has been generated with a temporal sampling frequency corresponding to the time step used in the LES computation. Moreover, compared to the spatial resolution of the computation, the PIV resolution was the same in the $\mathrm{y}$-direction and twice finner in the $\mathrm{z}$-direction. Thus, no spatial interpolation of the PIV data was performed. As the data are directly generated onto the computational mesh, the LES filtering is implicitly taken into account. Oneand two-point statistics of these velocity fields are presented in this section.

\section{One-point statistics}

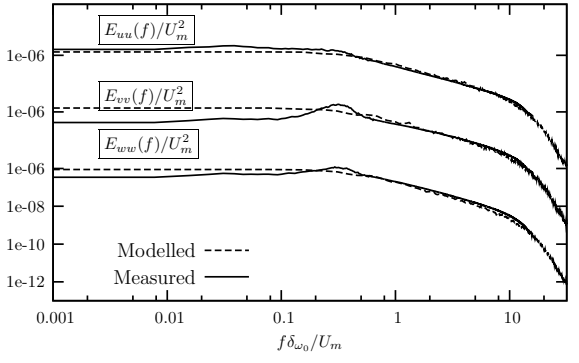

Figure 5: Spectra of the generated velocity fields obtained at $y / \delta_{\omega_{0}}=0.5$. - , hot-wire measurements; - - -, modelled data.
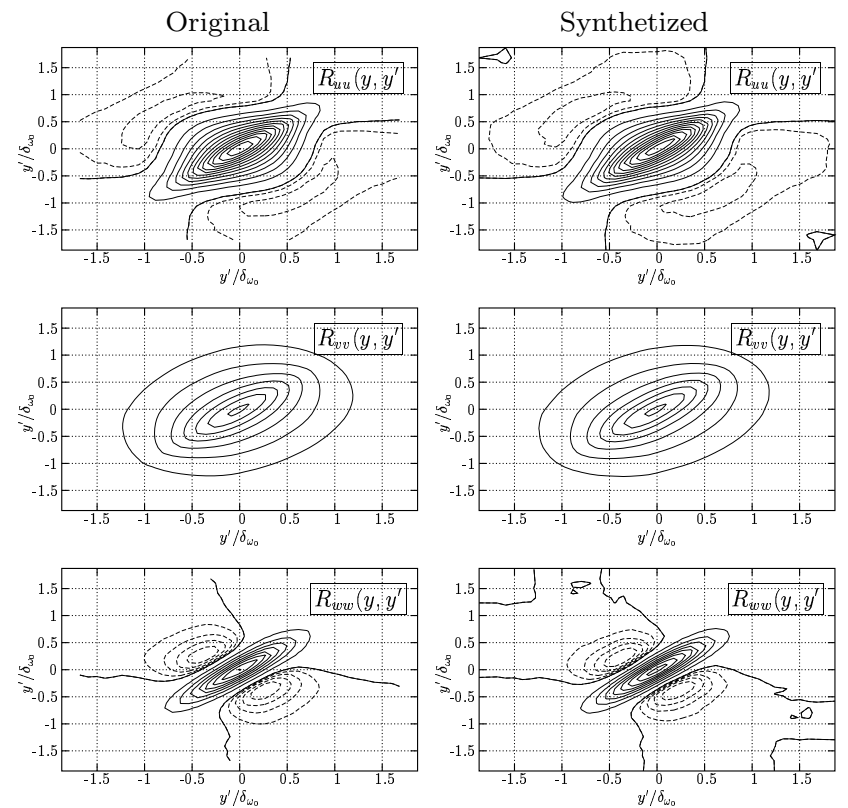

Figure 6: Two-point correlation $R_{i i}\left(y, y^{\prime}\right)$ of the original velocity fields (left) and synthetic velocity fields (right). positive increment between contours of $+0.5,---$ : negative increment between contours of -0.1 .

Vertical distribution of the energy of each velocity component, integrated over $z$, is presented in Figure 4. When compared to the statistics of the original experimental data, synthetized velocity fields exhibit correct energy levels as well as spatial repartition. In particular, the anisotropy of the flow and the shear stress $\overline{u^{\prime} v^{\prime}}$ are well reproduced.

Nonetheless, given the simple spectral model used for the temporal coefficients $a^{(n)}(t)$, typical frequencies of the mixing layer found by hot-wire measurements are not present in the generated data (Figure 5).

\section{Two-point statistics}

Figure 6 shows good agreement between measured and synthetized two-point correlations $R_{i i}\left(y, y^{\prime}\right)$. It must be stressed that, correlation levels, correlation lengths as well as the presence of regions of negative excursions are correctly reproduced by the proposed modelling approach.

In the spanwise direction (Figure 7), the two-point correlation tensor $R_{i i}\left(y, \delta_{z}\right)$ is also correctly reproduced. Never- 

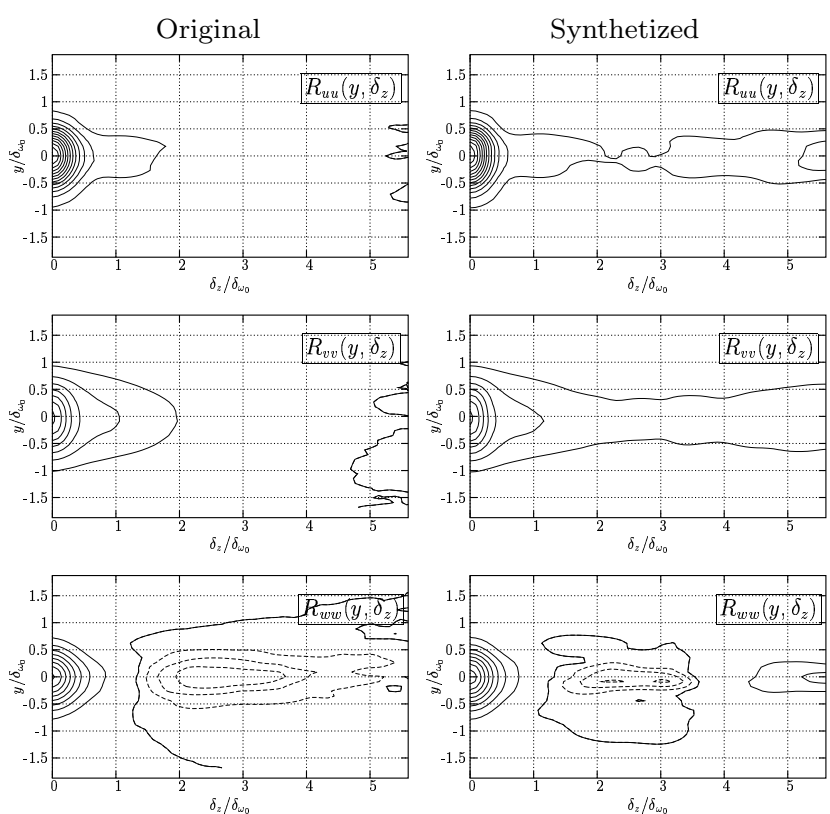

Figure 7: Two-point correlation $R_{i i}\left(y, \delta_{z}\right)$ of the original velocity fields (left) and synthetic velocity fields (right). positive increment between contours of +0.5 , - - -: negative increment between contours of -0.1 .

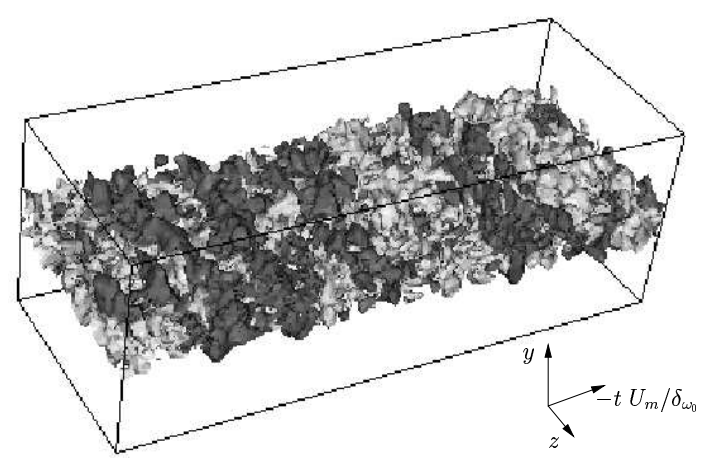

Figure 8: Isosurface of the vertical velocity component, $v \pm$ $0.02 U_{m}$ of the temporal evolution of the synthetic inflow data $\left(x=-U_{m} t\right)$.

theless, it appears that the extrapolation procedure affects the long range correlation levels which remain higher than the measured one. These differences can results from the introduction of parasite low wave numbers during the extrapolationperiodisation step.

\section{Instantaneous flow fields}

Temporal evolution of the generated velocity field in the inlet section (Figure 8) exhibits well organized regions both in the vertical and the spanwise directions. The random character of the generated time series leads to a lack of structuration in the temporal direction.

\section{LES WITH GENERATED INFLOW CONDITIONS}

A LES of the mixing layer is performed with Code_Saturne

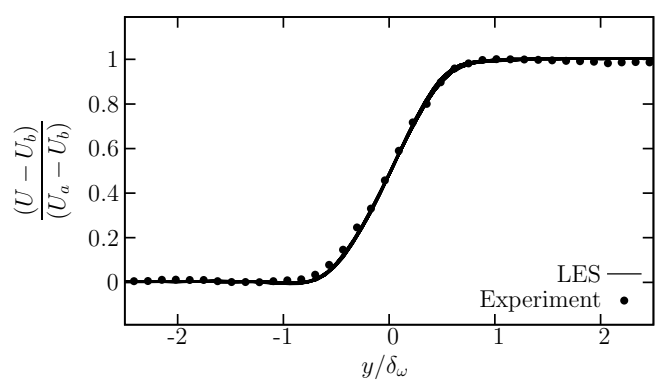

Figure 9: Longitudinal mean velocity profiles obtained by LES (lines) at different downstream locations (80 profiles superimposed for $\left.0<\left(x-x_{0}\right) / \delta_{\omega_{0}}<23\right)$ and by hot wire measurements (symbols), plotted in self-similar coodinates.

a finite volume solver on unstructured grids developed at EDF, for vectorial and parallel computing (Archambeau et al., 2004). The filtered, incompressible Navier-Stokes equations are solved with the Piomelli and Liu (1995) subgrid scale model. Space discretization is based on a collocation of all the variables at the center of gravity of the cells, using central differencing. Time discretization is based on a second order Crank-Nicolson/Adams-Bashforth method. The velocity/pressure system is solved by a SIMPLEC algorithm, with the Rhie and Chow interpolation. The Poisson equation is solved using a conjugate gradient method, with diagonal preconditioning. Computations are performed using 8 processors of a PC cluster. The inlet of the computational domain corresponds to the measurement section and the above-presented generated data are used as inlet conditions. The Reynolds number in the inlet section of the computation corresponds to the experimental one and is 36,000 . The spatial extent of the domain is $L_{x} \times L_{y} \times L_{z}=30 \delta_{\omega_{0}} \times 10 \delta_{\omega_{0}} \times 11 \delta_{\omega_{0}}$, meshed non regularly with $N_{x} \times N_{y} \times N_{z}=112 \times 53 \times 65$ points. The streamwise extent of the computational domain is chosen to enable the simulation of a significative evolution of the flow, corresponding to the doubling of the shear layer thickness $\left(\left(x-x_{0}\right) / \delta_{\omega_{0}} \leq 23\right)$. The last part of the domain is used to avoid some possible influence of the outflow condition.

The obtained LES results are here directly compared to those from the experiment of Perret (2004) performed at the same Reynolds number. Concerning the evolution of the simulated mean flow, it is seen in Figure 9 that the mean velocity profiles match the experimental one in the whole domain. The growth rate of the mixing layer is then correctly reproduced.

Study of the spatial repartition of the kinetic turbulent energy $\bar{k}=\frac{1}{2} \overline{\left(u^{\prime 2}+v^{\prime 2}+w^{\prime 2}\right)}$ and the shear stress $\overline{u^{\prime} v^{\prime}}$ across the shear layer (Figure 10) reveals good agreement with the experimental data, except in the region just downstream the inlet section where a decrease of $\bar{k}$ can be seen. It traduces the fact that the code must regenerate the correct phase information between modes to produce realistic turbulent structures. This drawback is common to all the methods based on the use of synthetic turbulence (see Keating et al., 2004 for a detailed review).

Results obtained with the present method are compared (Figure 10) to those obtained via hot-wire measurements at different downstream locations (Perret, 2004). It should be pointed out here that the inlet section is located in a region where the turbulent quantities are out of similarity regime, 
Table 1: Comparison of the fraction of reproduced Reynolds-shear stress and turbulent kinetic energy obtained by Druault et al. (2004) and in the present study, at two downstream locations.

\begin{tabular}{|c|c|c|c|c|c|c|}
\hline \multirow[b]{2}{*}{$\frac{x-x_{0}}{\delta_{\omega_{0}}}$} & \multicolumn{2}{|c|}{$\begin{array}{c}\text { Druault et al. } \\
\text { random }\end{array}$} & \multicolumn{2}{|c|}{$\begin{array}{l}\text { Druault et al. } \\
\text { realistic }\end{array}$} & \multicolumn{2}{|c|}{$\begin{array}{l}\text { present } \\
\text { study }\end{array}$} \\
\hline & $\overline{\overline{u^{\prime} v^{\prime}}}$ & $\bar{k}$ & $\overline{\overline{u^{\prime} v^{\prime}}}$ & $\bar{k}$ & $\overline{\overline{u^{\prime} v^{\prime}}}$ & $\bar{k}$ \\
\hline 5 & $5 \%$ & $3.9 \%$ & $110 \%$ & $88 \%$ & $86 \%$ & $64 \%$ \\
\hline 15 & $5 \%$ & $5.4 \%$ & $110 \%$ & $98.4 \%$ & $121 \%$ & $107 \%$ \\
\hline
\end{tabular}

a) Turbulent kinetci energy $\bar{k}$

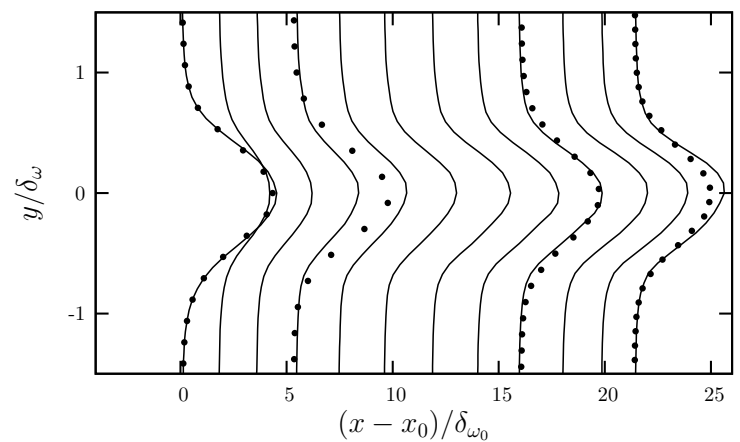

b) Reynolds shear stress $\overline{u^{\prime} v^{\prime}}$

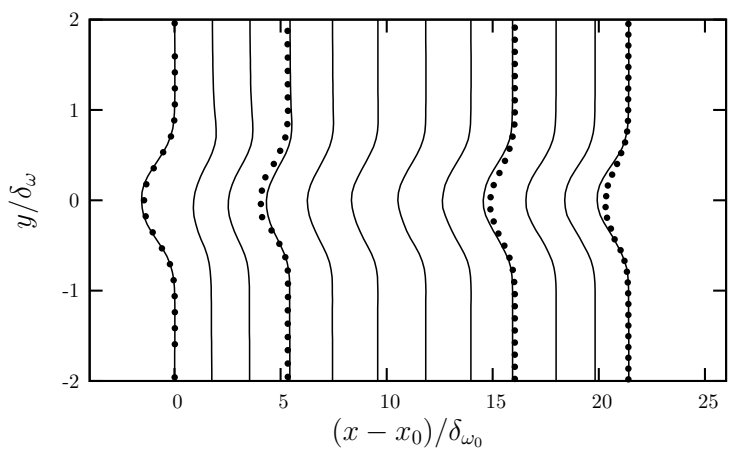

Figure 10: Downstream evolution of the profiles of a): $\bar{k}$ and b): $\overline{u^{\prime} v^{\prime}}$ plotted in self-similar coordinates. Lines: LES; symbols: hot wires experiment.

contrary to the mean flow. Hence, due to the presence of a region of adaptation just dowstream the inlet section, the code fails to correctly reproduce the slow decay of energy to the self-similarity level. However, the energy level obtained in the self-similarity region is reached approximately at the same spatial location both in the experiment and the computation. It should be noted that the use of simple Gaussian random fluctuations would have lead to an immediate laminarization of the flow (Druault et al., 2004, Keating et al., 2004). Thus, despite the decrease of the values of the turbulent quantities downstream of the inlet section due to the missing phase information, the proposed method enables the rapid development of the turbulence, by providing turbulence with proper length scales at the inlet. To illustrate this point, comparisons of the present results to the results obtained by Druault et al. (2004) are provided in table 1. These authors performed LES

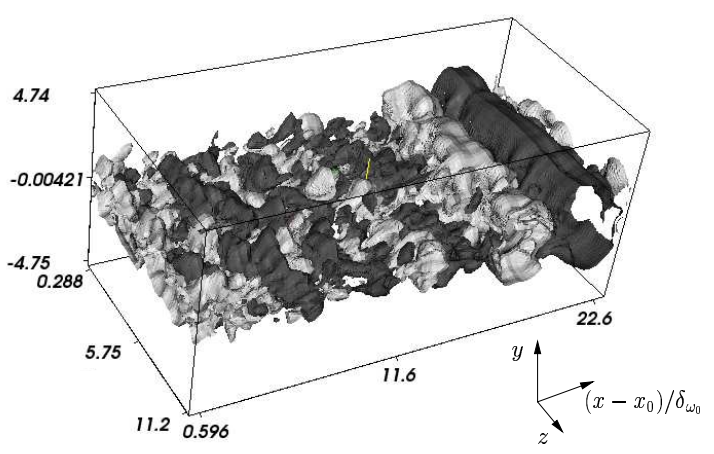

Figure 11: Isosurface of the vertical velocity component, $v \pm$ $0.02 U_{m}$ of the LES results.

of a turbulent mixing layer ( $R e \simeq 50,000)$ by using as inflow conditions, either random time series respecting only the 1-point statistics of the flow (referred to as random) or realistic velocity signals derived from an experimental database acquired by hot-wire measurement (referred to as realistic). It confirms that, if the realistic inflow conditions containing the realistic phase information (realistic case) lead to the best results, the present method performs very well compared to the use of purely random time series (random case).

Instantaneous simulated flow field (Figure 11) shows the rapid regeneration of realistic coherent structures with the downstream location.

\section{CONCLUSIONS}

An original approach to generate synthetic inflow conditions for numerical simulations has been presented and its viability has been demonstrated by performing a LES of a fully turbulent plane mixing layer. The proposed method is designed to interface stereoscopic PIV measurements, which are under-resolved in time, to a computional code via the use of the POD. This technique enables to perform separately the spatial adataption of the experimental data to the numerical mesh and the temporal modelling of the dynamics of the flow in the inlet section. Moreover, the one- and two-point moments corresponding to the targetted flow are directly prescribed to the time series, avoiding any modelling of these statistics.

Thus, the proposed method turns out to be able to generate synthetic data which present correct one- and two-point spatial statistics in the whole section as well as realistic turbulent spectra. Given the random character of the temporal 
series generated, LES using these data demonstrates the good performance of the proposed approach. Indeed, a turbulent mixing layer can be simulated by starting from a section where the flow is fully developed and highly turbulent.

Future work will concern the improvement of the temporal modelling of each POD modes to be able to take into account characteristic frequencies of the flow under interest. Strategies based on low-order dynamical system will be developed to model the dynamics of the first POD modes representating the large scale organization of the flow in the measurement section. Thus, by taking into account the correct phase information between the most energetic structures of the flow, the method is expected to reduce the adaptation region that exists downstream of the inlet section.

\section{ACKNOWLEDGEMENTS}

Financial support from the French Ministry of Defense is greatfully acknowledged. The authors also wish to thank P. Braud, C. Fourment and Dr. L. David for their contribution to the experimental part of this work.

\section{REFERENCES}

Archambeau, F., Mehitoua, N., and Sakiz, M., 2004, "Code Saturne: a Finite Volume Code for the Computation of Turbulent Incompressible flows", Int. J. Finite Volumes, Electronical edition: http://averoes.math.univ-paris13.fr/html

Berkooz, G., Holmes, P., and Lumley, J.L., 1993, "The Proper Orthogonal Decomposition in the analysis of turbulent flows", Annual Review of Fluid Mech., Vol. 25, pp. 539-575.

Coiffet, F., Delville, J., and Lamballais, E., 2002 "DNS/LES of a turbulent mixing layer using instantaneous experimental data", Advances in Turbulence IX. Proceedings of the Ninth European Turbulence Conference, Castro, I.P., Hancock, P.E., and Thomas, T.G., eds.

Druault, P., and Delville, J., 2000, "Representation of the spatial correlation tensor of the velocity in free turbulent flows", C.R. Acad. Sci. Paris, Série IIb, Vol. 328, pp. 135141.

Druault, P., Lardeau, S., Bonnet, J.P., Coiffet, F., Delville, J., Lamballais, E., Largeau, J.F., and Perret, L., 2004, "A Methodology for the Generation of Realistic 3D Turbulent Unsteady Inlet Conditions for LES", AIAA J., Vol. 42, pp. 447-456.

Franke, U., 1987, "Selective deconvolution: a new approach to extrapolation and spectral analysis of discrete signals", Int. Conf. on Acoustics, Speech Signal Processing. IEEE, May, pp. 30.3.1-30.3.4

Hinze, J.O., 1975, "Turbulence, 2nd ed", Mc Graw-Hill, New York.

Keating, A., Piomelli, U., Balaras, E., and Kaltenbach, H.J., 2004, "A priori and a posteriori tests of inflow conditions for large-eddy simulation", Phys. Fluids, Vol. 16, pp. 46964712.

Lee, S., Lele, S.K., Moin, P., 1992, "Simulation of spatially evolving turbulence and the applicability of Taylor's hypothesis in compressible flow", Phys. Fluids, Vol. 4, pp. 1521-1530.

Li, N., Balaras, E., and Piomelli, U., 2000, "Inflow conditions for large-eddy simulations of mixing layers", Phys. Fluids, Vol. 12, pp. 935-938.

Lumley, J.L., 1967, "The Structure of Inhomogeneous Turbulent Flows", Atm. Turb. and RadioWave Prop., Yaglom and Tatarsky eds., Nauka, Moscow, pp. 166-178.

Lund, T.S., Wu, X., and Squires, K.D., 1998, "Generation of turbulent inflow data for spatially-developing boundary layer simulations", J. Comput. Phys., Vol. 140, pp. 233-258.

Perret, L., 2004, "Etude du couplage instationnaire calculsexpérience en écoulements turbulents ", Ph.D. Thesis, Poitiers University, France.

Perret, L., Braud, P., Fourment, C., David, L. and Delville, J., 2004, "Mesure du champ d'accélération par vélocimétrie par imagerie de particules stéréoscopique deux plans", Proceedings of the gème Congrès Francophone de Vélocimétrie Laser, Bruxelles, Belgique.

Piomelli, U., and Liu, J., 1995, "Large-eddy simulation of rotating channel flows using a localized dynamic model", Phys. Fluids, Vol. 7, pp. 839-848.

Sirovich, L., 1987, "Turbulence and the dynamics of coherent structures. Part I: coherent structures", Quarterly of Applied Mathematics, XLV, Vol 3, pp. 561-571 